\title{
A legend of the SNARE complex and synaptotagmin - the insight into synaptic transmission
}

\author{
SUN JianYuan \\ Institute of Biophysics, Chinese Academy of Sciences, Beijing 100101, China
}

Received November 3, 2013; accepted November 4, 2013; published online November 11, 2013

Citation: Sun J Y. A legend of the SNARE complex and synaptotagmin—the insight into synaptic transmission. Sci China Life Sci, 2013, 56: 1150-1153, doi: $10.1007 / \mathrm{s} 11427-013-4572-3$

The 2013 Nobel Prize in Physiology or Medicine honors three scientists, James Rothman, Randy Schekman and Thomas Südhof, whose work revealed the mystery of the vesicle trafficking, i.e., how cells, from yeast to mammals, organize their transport systems. In a neuroscience perspective, this decades-long story might be described as a legend of identifying the SNARE complex and synaptotagmin proteins, revealing their functional mechanisms, and shedding an insight into synaptic transmission. Their research unmasked the exquisitely precise control of synaptic vesicle fusion. Here I will only review the history of how their pioneer work on vesicle fusion machinery served as a major step forward in our understanding of neuronal signaling.

Classical physiological studies revealed the central importance of synapses in brain function, indicating that neurons communicate between each other primarily by chemical transmission at the synapse. Synaptic function is crucial for the brain to mediate intelligence, feelings and behavior. Most neuronal and mental diseases such as schizophrenia, depression, bipolar disorder and many other pathological conditions attribute to synaptic defects. Through their discoveries, three scientists greatly advanced our understanding of neuronal information processing.

However, it is not an overnight thing-as Rothman described their achievements-most of it has been accomplished and developed over many years, if not decades. Indeed, it is a legend with many trials and tribulations. This actually was a decades-long legend of a group of scientists

*Corresponding author (email: jysun@ibp.ac.cn) who have extraordinary courage, determination, vision, and insight, in searching for this synaptic vesicle fusion machinery.

In mid-1970s, the general picture drawn by the pioneers of cell biology was that cells bustle with transport activities, multiple types of small bubble-like organelles called vesicles ferry cellular substances from one site to another, possibly via well-designated targeting pathways [1]. Some vesicles that shuttle between organelles had been revealed and even mapped in the pathway of membrane budding or fusing [1-3]. In the neuroscience field, it was found that nerve cells (or neurons) communicate with each other through specialized contact points called synapses. The synapse allows a neuron to transmit an electrical or chemical signal to another cell. As early as 1951, Bernard Katz demonstrated that a chemical transmitter is released from a structure at the end of the neuron, called the presynaptic nerve terminal, to the outside of the neuron in a quantized fashion [4,5]. The quantum-like signal was later found to be due to the secretion of multiple neurotransmitter molecules from a packet organelle called the "synaptic vesicle" in the nerve terminal [1]. Bernard Katz's another finding was that neurotransmitter releasing is highly regulated in response to $\mathrm{Ca}^{2+}$ influx into the presynaptic terminal [6]. This influx occurs when $\mathrm{Ca}^{2+}$ channels open in response to an electrical signal, called the action potential that is conducted to the nerve terminal [7]. For this work, Bernard Katz and George Palade were awarded the Nobel Prize one after another in 1970 and 1974. However, no one ever understood how vesicles budded from membranes; how vesicles knew their targets; and how 
vesicles were fused with their target membranes. These questions were complex and challenging for most cell biologists and neuroscientists, but to the young and fearless scientists, James Rothman, Randy Schekman and Thomas Südhof, the questions seemed to be more captivating because they never lacked ideas and courage to try something new and figure it out.

Both Rothman and Schekman decided to start with simple system as junior faculties in the late 1970s. Rothman tried to decompose the pathway by reducing vesicular transport to a set of elementary biochemical reactions and reconstitute it from the minimum number of components for budding and fusion in a test tube, while Schekman selected the way of genetic screening on the yeast Saccharomyces cerevisiae to obtain mutants that exhibit dysfunction in vesicle transport $[8,9]$. When they tried to track down the molecular machinery that performs the crucial trafficking functions, most of their colleague scientists were skeptical. Schekman, a biochemist himself, proposed to use yeast genetics to identify cellular trafficking components but he had no experience with yeast; Rothman tried to reconstitute from the minimum number of components of the budding and fusion machinery in a test tube by ignoring the complicated, multi-step biological processes, including those involved in DNA transcription and protein synthesis as part of the pathway. Furthermore, most scientists were arguing that breaking open the cells would destroy the spatial relationships, and the appropriate membranes might never restore their relative positions in the cells. People also questioned whether yeast had a robust secretion system and whether findings from such a single-celled creature were applicable to animals, especially to the mammalian central nervous system.

Regardless of the argument, Schekman and his graduate student, Peter Novick started the first experiment. They used a temperature-sensitive mutation strategy-raising the temperature would make a protein nonfunctional-to screen out the key genes required for secretion. The initial logic of this strategy is simple: the vesicle transport system is crucial for yeast to survive, the mutation in the crucial trafficking genes would cause a lethal phenotype [10]. Using electron microscopy, Schekman found that the mutants enhanced perturbations in the transport pathway. Some, for example, accumulated vesicles that presumably failed to fuse with their target membranes [10]. The ease of genetic manipulation and a successful strategy gave rise to the identification of the first $23 \mathrm{key}$ intracellular trafficking related genes ("sec") including 13 genes relevant to membrane fusion, and led to the discovery of an array of novel proteins involved in the secretory pathway, thus providing new insights into the molecular machinery that mediates vesicle budding and fusion [11].

Rothman's group used mutant mammalian cells and investigated vesicle trafficking by detecting the transport of a protein between membrane-bound compartments in a cell-free extract. Because people at that time were unable to express cloned genes in animal cells, they introduced the vesicular stomatitis virus (VSV) infection to mediate membrane protein expression and thus successfully built a test tube system for studying vesicle trafficking [12]. They mixed two homogenates: one of which contained a VSV-infected protein in a partially assembled form due to the lack of an essential transferase (the donor, made from mutant host cells); the other had no VSV infection but contained the radioactively tagged transferase (the acceptor, made from wild-type cells). If vesicles carrying the VSV-infected protein were to bud off the membrane from the donor homogenate, and then fused with the membrane from the acceptor homogenate, the radioactively tagged transferase could be incorporated into the proteins just arrived, completing the assembly - in this way, any membrane traffic could be monitored by detecting the destined radioactive protein in the test tube. This approach was soon proved to be successful and led to the finding of the fundamental principle, transport depends on the intrinsic chemical specificity of the membranes involved, rather than on the proximity of compartments [13]. Moreover, Rothman's group used this ingenious and powerful design to massively tease apart the proteins involved in vesicle budding and fusion [14].

A milestone in revealing the principle of membrane fusion is the identification of the SNARE complex. This story began in 1987 with the finding in Rothman's lab that cell-free transport is blocked by low concentrations of the sulfhydryl alkylating reagent $N$-ethylmaleimide (NEM). Then they purified the target of NEM and named it as $\mathrm{N}$-ethylmaleimide-sensitive factor (NSF) based on its ability to rescue the NEM-poisoned reaction $[15,16]$. When Rothman tracked down the gene that encodes NSF and sequenced it, he found it was the mammalian homologue of a previously cloned yeast gene SEC18, one that Schekman had isolated in his earlier experiments [10,17]. It was the first convergence of genetics and biochemistry between these two independent groups, suggesting that NSF/Sec18p was required for fusion even in such distantly related creatures as yeast and mammals. This boasted victory convinced many skeptics that both approaches were able to generate valuable insights and that in general, natural evolution in secretion system seems to be inherently robust. Although fusion needs NSF, but this protein cannot promote that reaction on its own. Later, Rothman found that NSF is only a cytosolic protein that binds to membranes by means of the soluble NSF attachment protein (SNAP) and the SNAP gene turned out to resemble another one of Schekman's screened genes, SEC17, which was found to be important for vesicle fusion [18]. In order to deeply dig into how SNAP and NSF work, Rothman proposed to search for the membrane receptors of SNAP, which he called the SNAP receptor, or SNARE. Guided by Schekman's pioneer work of genetic screening, two SNARE components on the cytoplasmic membrane, SNAP-25/Sec9p and syntaxin-1/Sec5p were 
soon cloned and Rothman demonstrated that these proteins form a complex and he named it as t-SNARE [19].

In the late 1980s and independently, Südhof set out to unveil the molecular underpinnings of the synaptic vesicle fusion. He asked the following important questions to himself, as always, immediately after he set up his lab in the University of Texas Southwestern Medical Center: How do the synaptic vesicle and the plasma membrane fuse in a millisecond time scale during synaptic transmission? How does calcium trigger synaptic vesicle fusion? How is calcium influx localized to the released sites in order to enable the fast coupling of an action potential to transmitter release?

Südhof combined membrane protein biochemistry with molecular biology techniques to study synaptic vesicles and the key proteins and soon isolated the vesicle-associated membrane protein (Richard Scheller named it VAMP and obtained it from the electric organ of a marine ray a year ago and Rothman named it as v-SNARE) from mammalian brain and called it synaptobrevin [20]. Furthermore, Südhof, in collaboration with Reinhard Jahn, discovered that VEMP/synaptobrevin/sec22p, SNAP-25/Sec9p and syntaxin-1/Sec5p are the substrates for the proteolytic activities of botulinum and tetanus toxins, which were known to block neurotransmitter release $[21,22]$. The identification of these three SNARE components and the relevant functional analysis provided the most important insight into the principle of membrane fusion, for which Rothman summarized as follows: the t-SNARE and v-SNARE components wrap around each other, thus to pull together the membranes in which they are embedded, thus prompting fusion [19].

As a neuroscientist, Südhof was fascinated by the ultrafast synaptic vesicle fusion which dictates precise neural signaling. Classical physiological work revealed the central importance of synapses in brain function, and characterized the mechanisms involved in synaptic transmission. He thought that Bernard Katz's discovery should not be a brilliant finale, but an opening of a revolutionary era of neuroscience-the era to elucidate how synapses work at the molecular level. In the line of calcium-dependent quantal release proposed by Katz, a delay in a microsecond time scale between calcium influx and neurotransmitter release would not allow the occurring of a cascade of protein-protein interactions. Therefore, there should be only a small number of molecules, or possibly a single molecule, to sense and trigger the fusion.

The cloning of a vesicle-associated protein called synaptotagmin-1 (syt-1) by Südhof and Jahn in 1990 was the turning point of the research [23]. Initially, Syt-1 was thought to be an evolutionarily conserved transmembrane protein with two cytoplasmic $\mathrm{C}_{2}$ domains, which represent the 'second constant sequence' in protein-kinase $\mathrm{C}$ isozymes [24]. However, it was later found to bind to phospholipids and perform calcium-dependent activities [23]. More importantly, Syt $1 \mathrm{C}_{2}$ domains can also bind to syntaxin-1 and the SNARE complex [25]. The new discovery of biochemical properties of Syt1 lead Südhof to speculate that syt-1 and/or some other protein family members might be the long-sought calcium sensor(s) for neurotransmitter release [26]. He kept decades-long focus on the systematic study of synaptotagmin protein family thereafter [27]. He carried out a series of biochemical studies on interactions between syt-1 and an array of presynaptic proteins [28]. In collaboration with structural biologists, he obtained the atomic structures of calcium-free and calcium-bound wild type and mutant Syt- $1 \mathrm{C}_{2}$ domains and provided the first insight into how the $C_{2}$ domains bind to calcium [29]. Suidhof pioneered the use of genetically modified mice to examine the functions of these key proteins and the machinery that regulates the calcium-dependent neurotransmitter releasing. His lab created a large number of genetically modified mice with the genes encoding synaptotagmins being knocked out or mutated and systematically revealed several important evidences. The in vivo study in his lab demonstrated that Syt1 knockout abolished the fast synchronous transmitter release and the mutation that changes the calcium affinity of endogenous Syt1 also altered the calcium-dependent synchronous neurotransmitter release to the same extent [30-32]. These conclusions were reached based on the fact that the synaptotagmin knock-out/mutations caused neither impairment in calcium influx into the nerve terminal nor the disturbance of the spatial coupling between calcium channels and vesicles [32]. Furthermore, they found that only three synaptotagmins-Syt1, Syt2 and Syt9-mediate fast synaptic vesicle exocytosis. Most forebrain neurons express only Syt1, whereas Syt2 is the predominant calcium sensor of very fast synapses in the brainstem, and Syt9 is primarily present in the limbic system. Only these isoforms of synaptotagmin were able to rescue the fast synchronous component of transmitter release in syt-1 deficient neurons [33]. All the results lead to the plausible mechanism that synaptotagmin functions as the calcium sensor and once calcium-binding sites of Synaptotagmin $\mathrm{C}_{2}$ domain are occupied, the calcium-activated synaptotagmin interacts with SNARE complex, thereby triggering synaptic vesicle fusion [32].

By ingeniously and elegantly designed experiments, these three scientists systematically exposed and analyzed the cascades of presynaptic protein-protein interactions involved in membrane fusion and gave an insight into the molecular mechanism that governs neurotransmitter release with precision. Nevertheless, many new questions now arise, which are not just details but of great importance. For example, what are the precise physicochemical mechanisms underlying the fusion process, and what defects of the fusion process would cause brain diseases? Much remains to be done in this field. A central goal of neuroscience is to understand the cell biology and biochemistry of the synapse, and to help us explain who we are, how we became who we are, and how we function moment by moment [34]. These three scientists' work made a great step forward to these 
goals, but their discovery should not be considered as a brilliant finale, but an opening of a new era of neuroscience. The legend of synapse study is still ongoing, people will further reveal the elaborate orchestrations underlying our sophisticated neurobiological activities, to understand the nature of thought and the identity of ourselves.

1 Palay S L, Palade G E. The fine structure of neurons. J Biophys Biochem Cytol, 1955, 1: 69-88

2 Claude A. Cell morphology and the organization of enzymatic systems in cytoplasm. Proc R Soc Lond B Biol Sci, 1954, 142: 177-186

3 De Duve C. The separation and characterization of subcellular particles. Harvey Lect, 1965, 59: 49-87

4 Fatt P, Katz B. An analysis of the end-plate potential recorded with an intracellular electrode. J Physiol, 1951, 115: 320-370

5 Fatt P, Katz B. Conduction of impulses in crustacean muscle fibres. J Physiol, 1951, 115: 45

6 Katz B, Miledi R. Ionic requirements of synaptic transmitter release. Nature, 1967, 215: 651

7 Hodgkin A L, Huxley A F. Resting and action potentials in single nerve fibres. J Physiol, 1945, 104: 176-195

8 Fries E, Rothman J E. Transport of vesicular stomatitis virus glycoprotein in a cell-free extract. Proc Natl Acad Sci USA, 1980, 77: 3870-3874

9 Novick P, Schekman R. Secretion and cell-surface growth are blocked in a temperature-sensitive mutant of Saccharomyces cerevisiae. Proc Natl Acad Sci USA, 1979, 76: 1858-1862

10 Novick P, Field C, Schekman R. Identification of 23 complementation groups required for post-translational events in the yeast secretory pathway. Cell, 1980, 21: 205-215

11 Schekman R, Novick P. 23 genes, 23 years later. Cell, 2004, 116: S13-S15, $1 \mathrm{p}$ following S19

12 Rothman J E, Fries E. Transport of newly synthesized vesicular stomatitis viral glycoprotein to purified Golgi membranes. J Cell Biol, 1981, 89: 162-168

13 Balch W E, Dunphy W G, Braell W A, et al. Reconstitution of the transport of protein between successive compartments of the Golgi measured by the coupled incorporation of $\mathrm{N}$-acetylglucosamine. Cell, 1984, 39: 405-416

14 Balch W E, Glick B S, Rothman J E. Sequential intermediates in the pathway of intercompartmental transport in a cell-free system. Cell, 1984, 39: 525-536

15 Block M R, Glick B S, Wilcox C A, et al. Purification of an N-ethylmaleimide-sensitive protein catalyzing vesicular transport. Proc Natl Acad Sci USA, 1988, 85: 7852-7856

16 Clary D O, Griff I C, Rothman J E. SNAPs, a family of NSF attachment proteins involved in intracellular membrane fusion in animals and yeast. Cell, 1990, 61: 709-721

17 Wilson D W, Wilcox C A, Flynn G C, et al. A fusion protein required for vesicle-mediated transport in both mammalian cells and yeast. Nature, 1989, 339: 355-359

18 Sollner $\mathrm{T}$, Whiteheart $\mathrm{S} \mathrm{W}$, Brunner $\mathrm{M}$, et al. SNAP receptors implicated in vesicle targeting and fusion. Nature, 1993, 362: 318-324

19 Weber T, Zemelman B V, McNew J A, et al. SNAREpins: Minimal machinery for membrane fusion. Cell, 1998, 92: 759-772

20 Sudhof T C, Baumert M, Perin M S, et al. A synaptic vesicle membrane protein is conserved from mammals to Drosophila. Neuron, 1989, 2: 1475-1481

21 Blasi J, Chapman E R, Link E, et al. Botulinum neurotoxin A selectively cleaves the synaptic protein SNAP-25. Nature, 1993, 365: $160-163$

22 McMahon H T, Ushkaryov Y A, Edelmann L, et al. Cellubrevin is a ubiquitous tetanus-toxin substrate homologous to a putative synaptic vesicle fusion protein. Nature, 1993, 364: 346-349

23 Perin M S, Johnston P A, Ozcelik T, et al. Structural and functional conservation of synaptotagmin (p65) in Drosophila and humans. J Biol Chem, 1991, 266: 615-622

24 Davletov B, Sontag J M, Hata Y, et al. Phosphorylation of synaptotagmin I by casein kinase II. J Biol Chem, 1993, 268: 6816-6822

25 Sudhof T C, Jahn R. Proteins of synaptic vesicles involved in exocytosis and membrane recycling. Neuron, 1991, 6: 665-677

26 Brose N, Petrenko A G, Südhof T C, et al. Synaptotagmin: A calcium sensor on the synaptic vesicle surface. Science, 1992, 256: 1021-1025

27 Pang Z P, Sudhof T C. Cell biology of $\mathrm{Ca}^{2+}$-triggered exocytosis. Curr Opin Cell Biol, 2010, 22: 496-505

28 Südhof T C. The synaptic vesicle cycle: A cascade of protein-protein interactions. Nature, 1995, 375: 645-653

29 Shao X, Fernandez I, Südhof T C, et al. Solution structures of the $\mathrm{Ca}^{2+}$-free and $\mathrm{Ca}^{2+}$-bound C2A domain of synaptotagmin I: Does $\mathrm{Ca}^{2+}$ induce a conformational change? Biochemistry, 1998, 37: 16106-16115

30 Fernandez-Chacon R, Königstorfer A, Gerber S H, et al. Synaptotagmin I functions as a calcium regulator of release probability. Nature, 2001, 410: 41-49

31 Geppert M, Goda Y, Hammer R E, et al. Synaptotagmin I: A major $\mathrm{Ca}^{2+}$ sensor for transmitter release at a central synapse. Cell, 1994, 79: 717-727

32 Sun J, Pang Z P, Qin D, et al. A dual- $\mathrm{Ca}^{2+}$-sensor model for neurotransmitter release in a central synapse. Nature, 2007, 450: 676-682

33 Xu J, Mashimo T, Südhof T C. Synaptotagmin-1, -2, and -9: Ca(2+) sensors for fast release that specify distinct presynaptic properties in subsets of neurons. Neuron, 2007, 54: 567-581

34 Südhof T C. A molecular machine for neurotransmitter release: Synaptotagmin and beyond. Nat Med, 2013, 19: 1227-1231

Open Access This article is distributed under the terms of the Creative Commons Attribution License which permits any use, distribution, and reproduction in any medium, provided the original author(s) and source are credited. 